\title{
Therapeutic Co-targeting of WEE1 and ATM Downregulates PD-L1 Expression in Pancreatic Cancer
}

Mei Hua Jin, MD, MS'1
Ah-Rong Nam, MS
Ji Eun Park, MS
Ju-Hee Bang, MS
Yung-Jue Bang, MD, PhD 1,2
Do-Youn Oh, MD, PhD

${ }^{1}$ Cancer Research Institute, Seoul National University College of Medicine, Seoul, ${ }^{2}$ Department of Internal Medicine, Seoul National University Hospital, Seoul, Korea

\begin{abstract}
Purpose
Pancreatic cancer (PC) is one of the most lethal cancers worldwide, but there are currently no effective treatments. The DNA damage response (DDR) is under investigation for the development of novel anti-cancer drugs. Since DNA repair pathway alterations have been found frequently in PC, the purpose of this study was to test the DDR-targeting strategy in PC using WEE1 and ATM inhibitors.
\end{abstract}

\section{Materials and Methods}

We performed in vitro experiments using a total of ten human PC cell lines to evaluate antitumor effect of AZD1775 (WEE1 inhibitor) alone or combination with AZD0156 (ATM inhibitor). We established Capan-1-mouse model for in vivo experiments to confirm our findings.

\section{Results}

In our research, we found that WEE1 inhibitor (AZD1775) as single agent showed anti-tumor effects in PC cells, however, targeting WEE1 upregulated p-ATM level. Here, we observed that co-targeting of WEE1 and ATM acted synergistically to reduce cell proliferation and migration, and to induce DNA damage in vitro. Notably, inhibition of WEE1 or WEE1/ATM downregulated programmed cell death ligand 1 expression by blocking glycogen synthase kinase-3 $\beta$ serine 9 phosphorylation and decrease of CMTM6 expression. In Capan-1 mouse xenograft model, AZD1775 plus AZD0156 (ATM inhibitor) treatment reduced tumor growth and downregulated tumor expression of programmed cell death ligand 1, CMTM6, CD163, and CXCR2, all of which contribute to tumor immune evasion.

\section{Conclusion}

Dual blockade of WEE1 and ATM might be a potential therapeutic strategy for PC. Taken together, our results support further clinical development of DDR-targeting strategies for PC.
Department of Internal Medicine,

Seoul National University College of Medicine, 101 Daehak-ro, Jongno-gu, Seoul 03080, Korea

Tel: 82-2-2072-0701

Fax: 82-2-762-9662

E-mail: ohdoyoun@snu.ac.kr

Published Online June 25, 2019

\section{Key words}

Pancreatic neoplasms, DNA damage response, WEE1, ATM, PD-L1

\section{Introduction}

Pancreatic cancer (PC) is one of the most lethal diseases worldwide, and there is an urgent need to develop effective therapies for this disease [1,2]. Recently, genomic analyses have revealed that many cancer susceptibility genes are frequently mutated in PC, including KRAS (92\% of cases) and TP53 (78\%). In addition, germline and somatic mutations in genes encoding breast cancer 1 and 2 (BRCA1, BRCA2), ataxia telangiectasia mutated (ATM) and partner and localizer of BRCA2 (PALB2) are present in PC patients with frequencies of $5 \%$ (germline mutations) and $12 \%$ (somatic mutations) [1]. These genes play critical roles in the DNA damage response (DDR), which signals the presence of strand breaks and other forms of DNA damage and coordinates their repair. Paradoxically, mutations in genes that compromise the DDR can both cause and protect against cancer. On the one hand, defects in the DDR can lead to genomic instability and the accumulation of mutations that increase the probability of 
cancer. On the other hand, DDR pathway dysfunction can render tumor cells susceptible to chemotherapeutic agents that damage DNA and / or impair alternative DDR pathways [3]. Thus, targeting of specific molecules in the DDR is a potential strategy for the development of new drugs for cancers with urgent unmet needs, including PC.

The cellular response to DNA damage, including singlestranded or double-stranded DNA breaks, is controlled by a network of proteins that include damage-sensing proteins such as poly(ADP-ribose) polymerase (PARP); transducers such as the kinases ATM and ataxia telangiectasia and Rad3 related (ATR); and effectors such as the nuclear kinase WEE1, which is a key protein in cell cycle checkpoint control and inhibits entry into mitosis [3]. Ultimately, the DDR results in one of three outcomes for the cell: successful DNA repair, cell cycle arrest, or apoptosis.

Therapeutic targeting of the DDR pathway has been examined in diverse tumor types [3]. Notably, inhibition of PARP, ATM, ATR, or WEE1 has been shown to abrogate DNA repair via homologous recombination (HR) in many cancers with a genetically defective DDR, thus leading to synthetic lethality [4-7]. WEE1 acts as a gatekeeper of the G2/M cell cycle checkpoint, and its activity increases during the $S$ and G2 phases; thus, WEE1 inhibition can induce growth arrest in $S$ phase [8]. In contrast, cancer cells expressing mutant TP53 lack a functional G1 checkpoint, and DNA damage must be repaired during the $\mathrm{G} 2 / \mathrm{M}$ transition. Given that TP53 is often mutated in PC [8], PC is the good candidate for the development of the DDR-acting agents.

The tumor microenvironment plays a critical role in cancer progression [9]. PC is unique compared with other tumor types in being surrounded by strong stroma. Abundant immunosuppressive cells reside in the tumor microenvironment, including regulatory $\mathrm{T}$ cells, myeloid-derived suppressor cells (MDSCs), M2-type macrophages, and cancer-associated fibroblasts (CAFs) [9,10]. Recruitment of these cells establishes a barrier to the anti-tumor immune response $[9,10]$. In addition, signaling via the chemokine receptor CXCR2 can drive PC growth by recruiting MDSCs and tumor-associated neutrophils and by enhancing the metastatic process [10]. Programmed cell death ligand 1 (PD-L1) is a negative regulator of the immune response and acts by binding to its receptor programmed cell death 1 (PD-1) on T cells, which inactivates the cells and thus allows the tumor to escape immune surveillance [11]. Data from genomic analyses indicate that immunogenic subtype of PC, which exhibits high levels of PD-L1, cytotoxic T-lymphocyte-associated protein 4 and CXCR2 among several subtypes [1]. Notably, the chemokine-like factor-like MARVEL transmembrane domain containing family member 6 (CMTM6) has been suggested as one of the mechanisms of regulation of PD-L1 through preventing PD-L1 degradation by lysosome [12].
Increasing evidence suggests the existence of crosstalk between the DDR signaling network and immune pathways $[13,14]$. For example, recent studies have demonstrated that the DDR regulates PD-L1 expression in cancer cells via a pathway involving activation of signal transducer and activator of transcription (STAT) signaling and inactivation of glycogen synthase kinase-3 $\beta$ (GSK-3 $\beta$ ) $[15,16]$. However, such interactions between the DDR and immune signaling have not yet been studied in PC.

Here, we evaluated the anti-tumor effects of targeting the DDR using a WEE1 kinase inhibitor (AZD1775) and an ATM kinase inhibitor (AZD0156) in PC cells in vitro and in a mouse xenograft model. We also examined the expression and activation of a number of DDR-related and immune signalingrelated molecules to identify potential crosstalk between the DDR and immune system in PC.

\section{Materials and Methods}

\section{Human cell lines and reagents}

Ten human PC cell lines were employed in this study: AsPC-1, Capan-1, Capan-2, MIA PaCa-2, PANC-1, SNU213, SNU324, and SNU410 were purchased from the Korean Cell Line Bank (Seoul, Korea), and SNU2913 and SNU2918, patient-derived cell lines, were successfully established from patient. Cells were cultured in medium (MIA PaCa-2 and PANC-1 cells in Dulbecco's modified Eagle's medium, all other cell lines in RPMI-1640, both from Welgen Inc., Gyeongsan, Korea) supplemented with 10\% fetal bovine serum and $10 \mu \mathrm{g} / \mathrm{mL}$ gentamicin and were maintained at $37^{\circ} \mathrm{C}$ in a $5 \%$ $\mathrm{CO}_{2}$ atmosphere. The WEE1 inhibitor AZD1775 and ATM inhibitor AZD0156 were kindly provided by AstraZeneca (Macclesfield, UK).

\section{PD-L1 expression analysis by flow cytometry}

Cells $\left(2 \times 10^{5}\right)$ were seeded in 60-mm dishes and incubated with AZD1775 and/or AZD0156 for 72 hours. The adherent cells were harvested, resuspended in cell staining buffer (\#420201, BioLegend, San Diego, CA), and incubated with anti-PD-L1 antibody (\#329708, BioLegend) for 30 minutes at room temperature. Cells were then washed once with the same buffer and analyzed on a FACSCalibur. The results are presented as the means of three independent experiments. 


\section{Human cytokine array}

Cells $\left(1 \times 10^{6}\right)$ were seeded in $60-\mathrm{mm}$ dishes and exposed to AZD1775 and/or AZD0156 for 24 hours. The cell supernatant was then collected and $500 \mu \mathrm{L} /$ sample was analyzed using the Proteome Profiler Human Cytokine Array Kit (\#ARY-005B, R\&D Systems, Minneapolis, MN) according to the manufacturer's instructions. Spot intensities were measured using ImageJ software (National Institutes of Health, Betheda, MD).

\section{Human phospho-kinase array}

Cells $\left(1 \times 10^{6}\right)$ were seeded in 100-mm dishes and exposed to AZD1775 and/or AZD0156 for 72 hours. The cells were harvested, and lysate samples containing $300 \mu \mathrm{g}$ of proteins were analyzed using the Proteome Profiler Human PhosphoKinase Array Kit (\#ARY003B, R\&D Systems) according to the manufacturer's instructions. Spot intensities measured using ImageJ software.

\section{Tumor xenograft experiments}

Four-week-old female athymic nude mice were purchased from Orient Bio Inc. (Seongnam, Korea). Capan-1 cells were resuspended at $3 \times 10^{7}$ cells in $100 \mu \mathrm{L}$ of phosphate-buffered saline and injected subcutaneously. The tumor volume was calculated using the formula: volume $=\left[(\text { width })^{2} \times\right.$ height $] / 2$. When the tumor volume reached $200 \mathrm{~mm}^{3}$, the mice were randomly assigned to four groups of five mice to receive (1) vehicle (2-hydroxypropyl- $\beta$-cyclodextrin solution), (2) AZD1775 once daily at $30 \mathrm{mg} / \mathrm{kg}$ for 4 weeks ( 5 days on $/ 2$ days off), (3) AZD0156, as described for (2), or (4) AZD1775 plus AZD0156, as described for (2). All treatments were administered by oral gavage. Body weights and tumor sizes were measured every other day.

\section{Mouse cytokine array}

Immediately before sacrifice, the mice were bled and serum samples were prepared. Aliquots of $500 \mu \mathrm{L}$ were analyzed using the Proteome Profiler Mouse Cytokine Array Kit, Panel A (\#ARY006, R\&D Systems) according to the manufacturer's instructions. Spot intensities were measured using ImageJ software.

\section{Statistical analysis}

Analyses were conducted using SigmaPlot version 10.0 (Systat Software Inc., San Jose, CA). Data are presented as the mean \pm standard errors. All statistical tests were twosided. Differences were considered significant if the p-values were $<0.05$. Half-maximal inhibitory concentrations $\left(\mathrm{IC}_{50}\right)$ were calculated using SigmaPlot software. Combined drug effects were analyzed by calculating the combination index (CI) with CalcuSyn software (Biosoft, Cambridge, UK). CI values of $<1,1$, and $>1$ indicate synergistic, additive, and antagonistic effects, respectively.

\section{Others}

Further information on cell viability assay, colony-forming assay, western blot analysis, immunoprecipitation, cell cycle analysis, Annexin V/ propidium iodide (PI) apoptosis assay, comet assay, transwell migration assay, and immunohistochemistry can be found in supplementary methods.

\section{Ethical statement}

Animal experiments were performed at the Biomedical Center for Animal Resource Development of Seoul National University (Seoul, Korea) according to institutional guidelines, and prior approval of the study protocol was obtained from the Institutional Animal Care and Use Committee.

\section{Results}

\section{WEE1 inhibition inhibits the proliferation of PC cells}

To evaluate the anti-tumor effects of WEE1 inhibition in PC, we used the MTT assay to assess the proliferation of 10 human PC cell lines in the presence of AZD1775 for 72 hours. As shown in Fig. 1A, AZD1775 inhibited the proliferation of all PC cell lines in a dose-dependent manner. The concentrations causing $50 \%$ inhibition $\left(\mathrm{IC}_{50}\right.$ ) ranged from 0.5 to $2.1 \mu \mathrm{M}$ (S1 Table). To verify these data, we examined the ability of the PC cell lines to form colonies after 10 days of incubation with AZD1775. This analysis also indicated a profound suppression of colony formation in all PC cell lines (Fig. 1B), and the low $\mathrm{IC}_{50}$ values for colony formation $(0.03-0.36 \mu \mathrm{M})$ confirmed the sensitivity of human PC cells to WEE1 inhibition (S1 Table). Because all 10 of the PC cell lines showed comparable inhibition by AZD1775, we randomly selected four cell lines (Capan-1, SNU213, SNU410, and SNU2913) for the following experiments.

To determine whether AZD1775 blocked signaling in the DDR pathway, we performed western blot analysis of the expression and activation (phosphorylation) of a number of molecules involved in DDR signaling downstream of WEE1. For these experiments, PC cells were incubated with or without AZD1775 for 24 hours before analysis by western blot- 

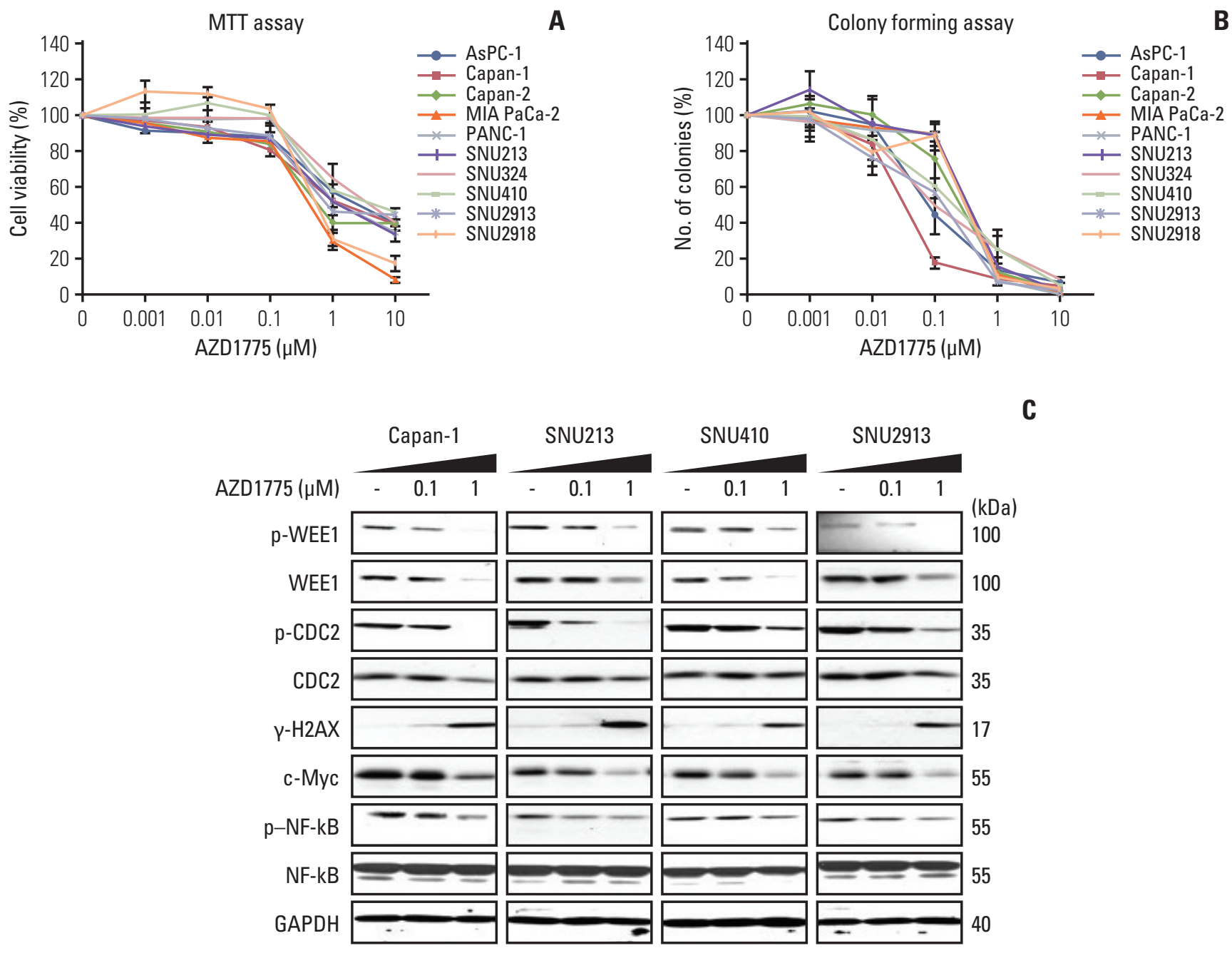

C

Fig. 1. Growth inhibitory effect of AZD1775 in pancreatic cancer (PC) cell lines. (A) Ten PC cell lines were exposed to control (0) or $0.001,0.01,0.1,1$, and $10 \mu \mathrm{M}$ AZD1775 for 72 hours, and cell viability was measured using the MTT assay. (B) Cell lines were treated as described in A and colony formation was analyzed after 10 days. (C) Western blot analysis of total or phosphorylated signaling molecules in Capan-1, SNU213, SNU410, and SNU2913 cells treated with 0, 0.1, or $1 \mu$ M AZD1775 for 24 hours. Experiments were repeated three times. GAPDH, glyceraldehyde 3-phosphate dehydrogenase; p-NF-kB, phosphorylated nuclear factor kappa-light-chain-enhancer of activated B cells. (Continued to the next page)

ting. As shown in Fig. 1C, we confirmed that AZD1775 dosedependently reduced the expression of total and phosphorylated WEE1 (p-WEE1) in all PC cells tested. In addition, AZD1775 also decreased the expression of phosphorylated cell division cycle protein 2, c-Myc, and phosphorylated nuclear factor kappa-light-chain-enhancer of activated B cells (p-NF-kB), and upregulated the expression of $\gamma$-H2AX compared with control (Fig. 1C). These results suggest that AZD1775 monotherapy has anti-proliferative activity in PC cells.

\section{WEE1 inhibition induces S-phase arrest and apoptosis in PC cells}

Since WEE1 inhibition reduced PC cell proliferation, we next determined whether AZD1775 induced apoptosis. For this, the cells were incubated with or without AZD1775 for 48 hours and apoptosis was examined by flow cytometry of Annexin V / PI-stained cells or by western blot analysis of an apoptosis regulator, MCL-1, and an effector, cleaved caspase 7. We found that AZD1775 treatment significantly increased the proportion of apoptotic cells compared with control cells 

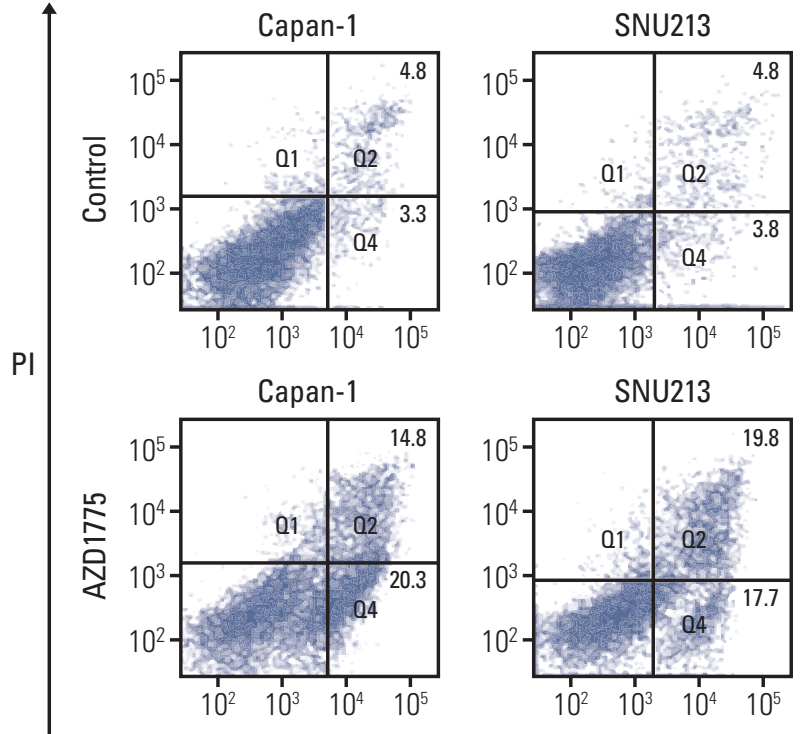
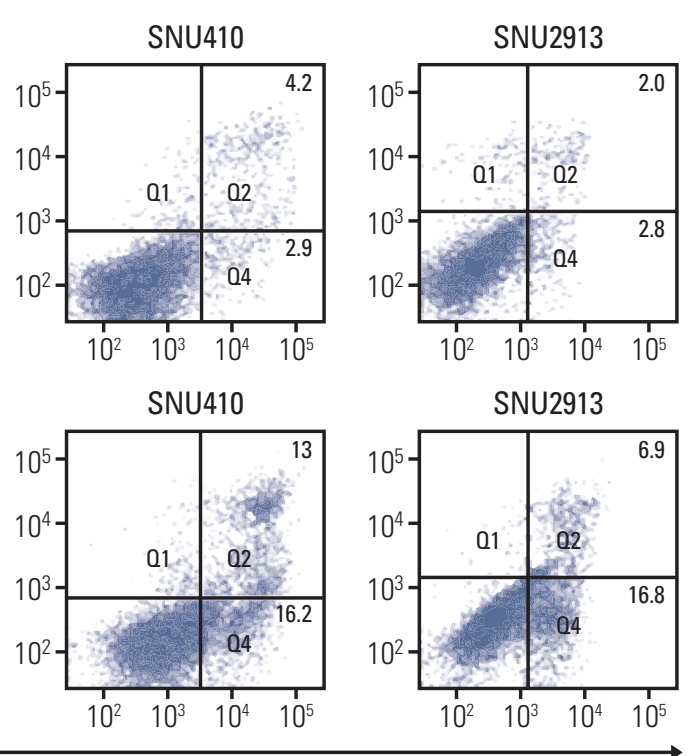

Annexin V
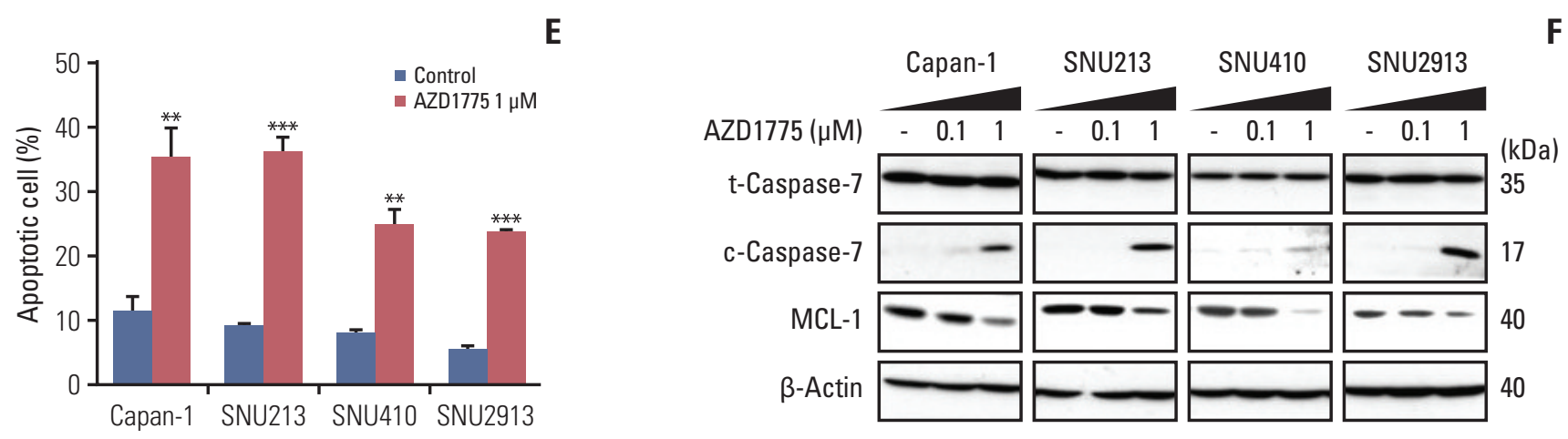

G
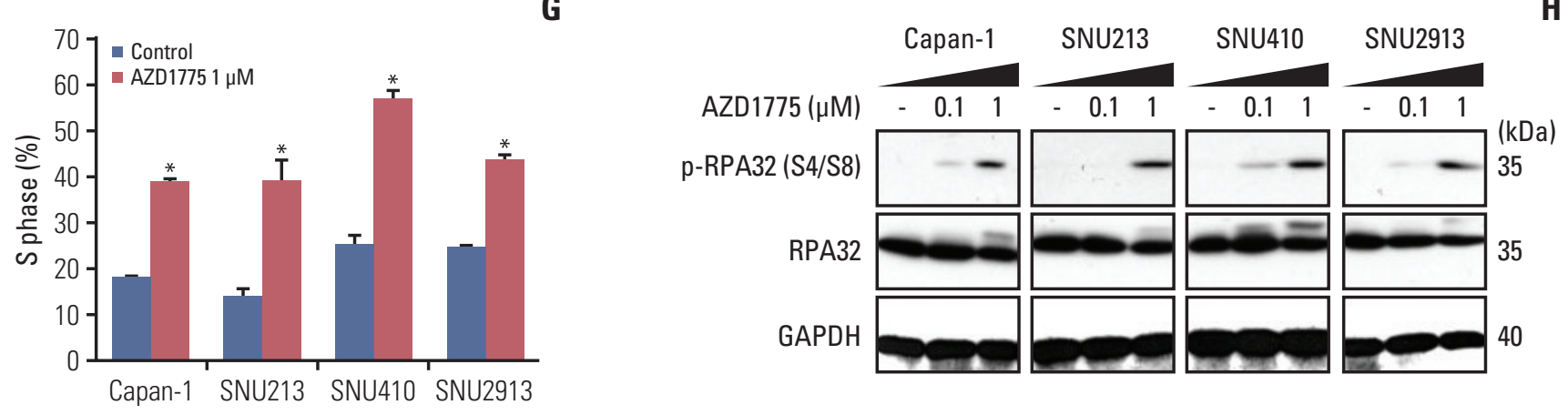

Fig. 1. (Continued from the previous page) (D) Annexin V/propidium iodide (PI) apoptosis assay of Capan-1, SNU213, SNU410, and SNU2913 cells treated with 0 or $1 \mu \mathrm{M}$ AZD1775 for 48 hours. (E) Quantification of three independent Annexin V/PI assays. (F) Western blot analysis of apoptosis-related proteins in cells treated with 0, 0.1, or $1 \mu \mathrm{M}$ AZD1775 for 48 hours. (G) Cell cycle analysis of PI-stained PC cells treated with 0 or $1 \mu \mathrm{M}$ AZD1775 for 24 hours. (H) Western blot analysis of cell cycle arrest-related signaling molecules in cells treated with $0,0.1$, or $1 \mu \mathrm{M}$ AZD1775 for 24 hours. ${ }^{*} \mathrm{p}<0.05,{ }^{* *} \mathrm{p}<0.01,{ }^{* * *} \mathrm{p}<$ 0.001. Experiments were repeated three times. 


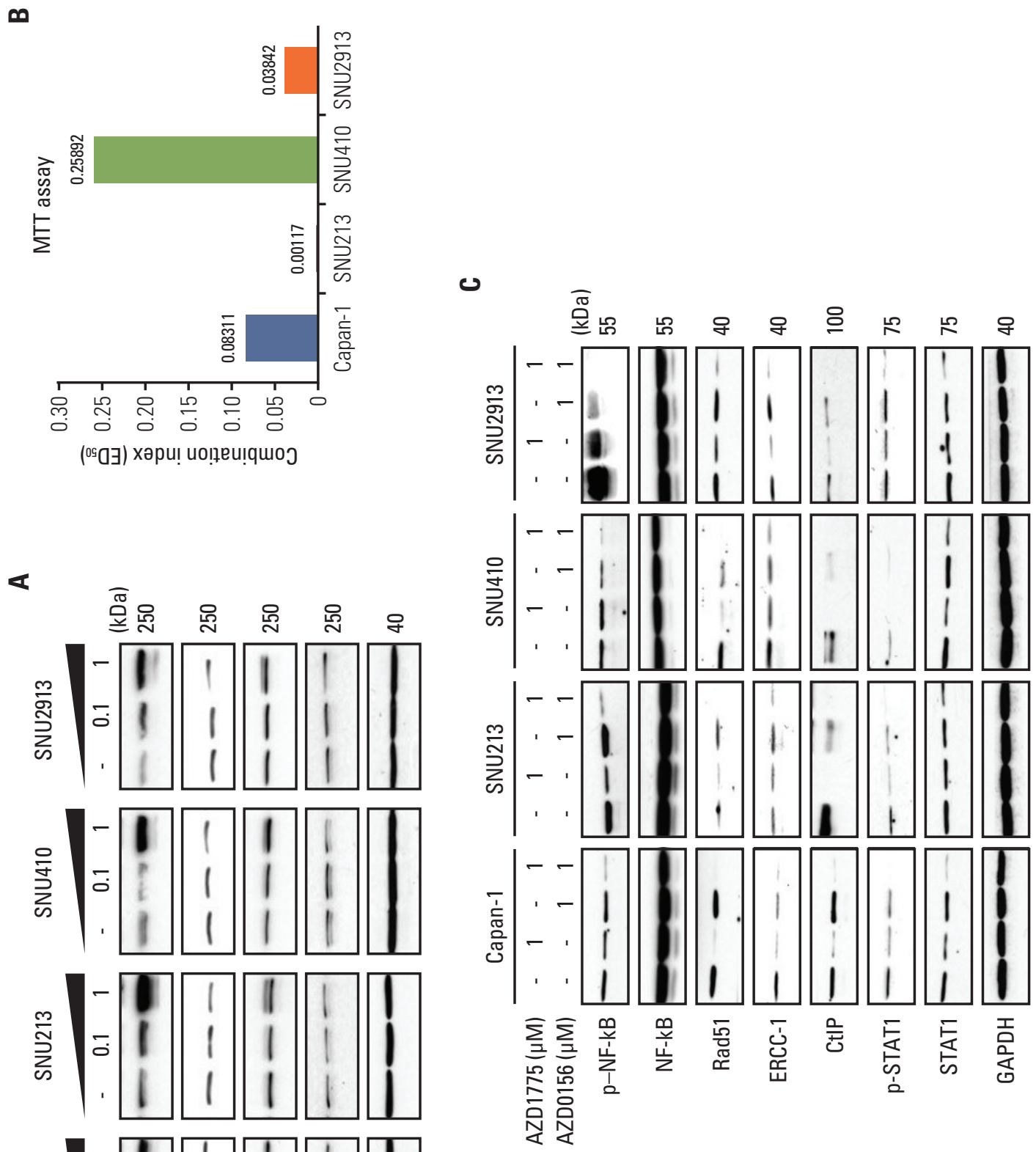

के

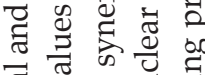
퓽 पै बे क

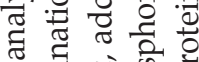
음 है 둔 0 क 包艺光 उ.

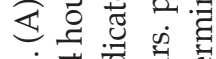

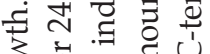
o $\overrightarrow{0} \vec{v}$ 要至导 곳슈 ๖ 施通

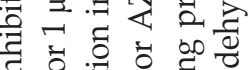

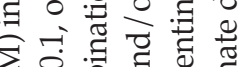

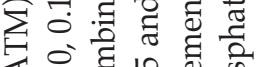
《宅企 के चु क्षे है

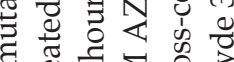
む

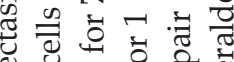
.

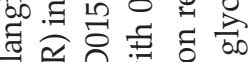

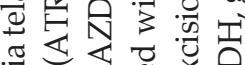
采芯芯

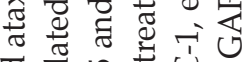

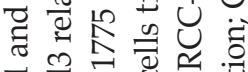

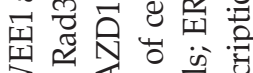

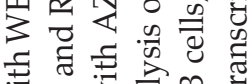

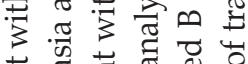

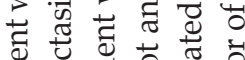
苟 ฮ્

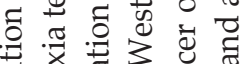

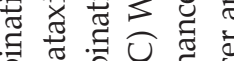
है

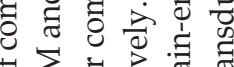

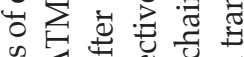

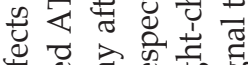

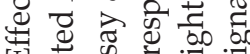

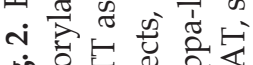

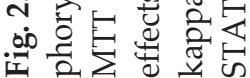


A
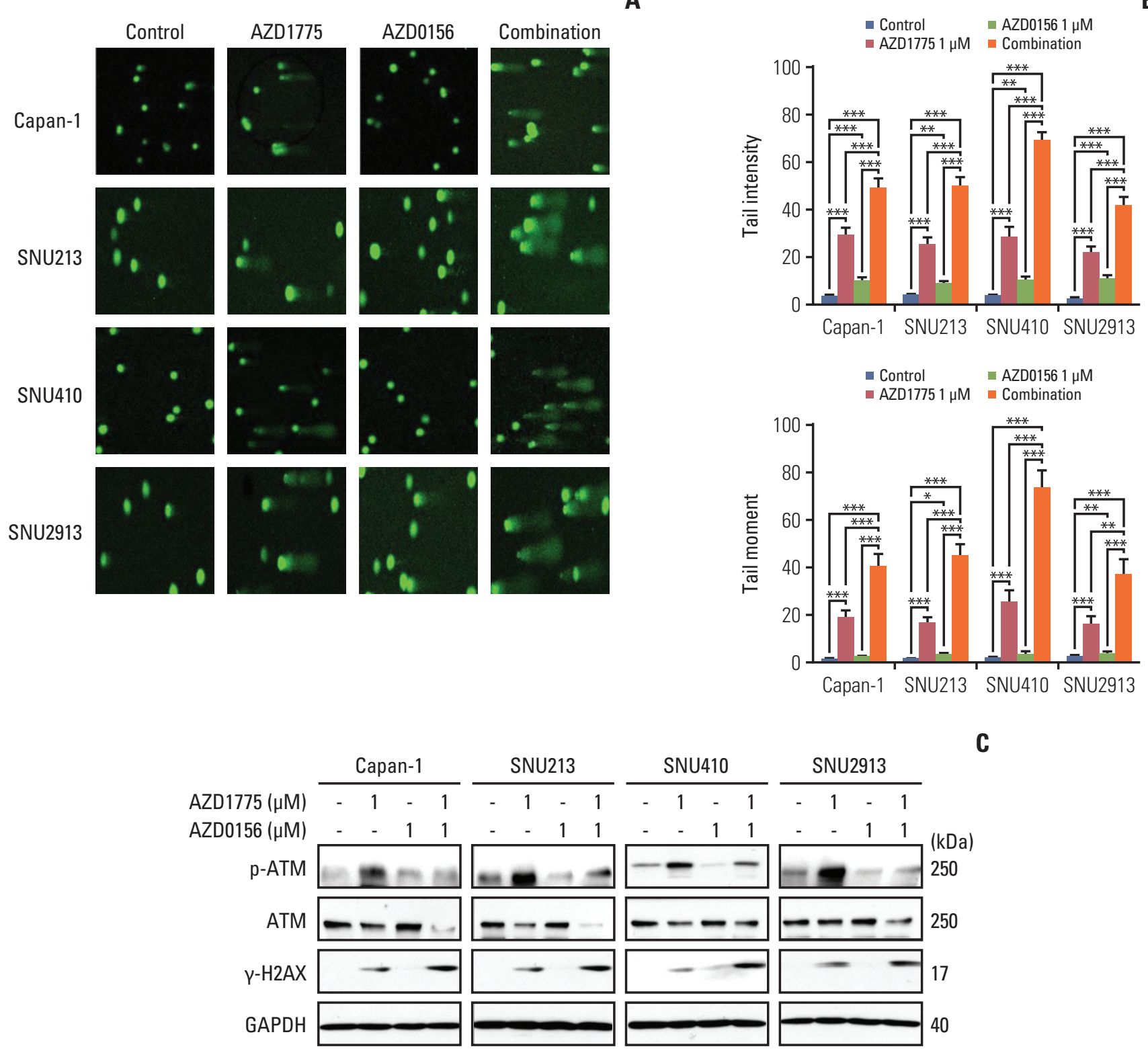

Fig. 3. Effect of WEE1 and ataxia telangiectasia mutated (ATM) co-inhibition on DNA damage. (A, B) Displayed Comet Assay results after treated with 0, AZD1775 (1 $\mu \mathrm{mol} / \mathrm{L})$ alone, AZD0156 (1 $\mu \mathrm{mol} / \mathrm{L})$ alone, or both for 24 hours. Tail intensity and moment were analyzed using the Comet Assay IV program. (C) The related DNA damage signals were detected after exposed at combination treatment setting for 24 hours. ${ }^{* *} \mathrm{p}<0.01$, ${ }^{* * *} \mathrm{p}<0.001$. Experiments were repeated three times. GAPDH, glyceraldehyde 3-phosphate dehydrogenase.

(Fig. 1D and E) and concomitantly diminished the expression of MCL-1 and elevated the expression of cleaved caspase-7 (Fig. 1F). Since WEE1 functions as a regulator of cell cycle progression, we also assessed the proportion of cells in the cell cycle phases by flow cytometric analysis of PI-stained cells. As shown in Fig. 1G, a significantly greater proportion of cells treated with AZD1775 than control was arrested in
S-phase. To verify this, we examined expression of phosphorylated replication protein A 32 (p-RPA32), an S-phase marker that binds to single-stranded DNA. Indeed, AZD1775 treatment for 24 hours resulted in increased p-RPA32 at serine 4 and 8, indicating that WEE1 inhibition leads to replication stress (Fig. 1H). 


\section{Co-inhibition of WEE1 and ATM profoundly impairs activation of the HR pathway of DNA repair}

Having evaluated the effects of AZD1775 as a single agent, we asked whether WEE1 inhibition influences the activity of other core members of the DDR, such as ATM or ATR. Indeed, we found that phosphorylation of ATM and ATR was increased in cells treated for 24 hours with AZD1775 (Fig. 2A). Since AZD1775 had a greater effect on promoting phosphorylation of ATM than of ATR, we next examined the anti-proliferative effects of AZD1775 in combination with the ATM inhibitor AZD0156 using the MTT assay. As shown in Fig. 2B, the CI values of AZD0156 plus AZD1775 were less than 1 for all four PC cell lines, indicating that the drugs had a synergistic effect on proliferation.

To better understand the effects of combination AZD1775 and AZD0156 treatment, we examined the expression levels of several molecules required for DNA repair via HR, after treatment of cells for 72 hours with AZD0156 and / or AZD1775. We found that expression of $\mathrm{p}-\mathrm{NF}-\mathrm{kB}$, Rad51, excision repair cross-complementing protein $1, \mathrm{C}$-terminal binding protein-interacting protein, and p-STAT1 were all downregulated by AZD1775 or AZD0156 when added alone, but a greater effect was observed in cells co-treated with both agents (Fig. 2C). These data suggest that co-inhibition of WEE1 and ATM strongly blocks the HR pathway of DNA repair.

\section{Co-inhibition of WEE1 and ATM synergistically induces DNA damage}

Having demonstrated that co-inhibition of WEE1 and ATM blocks DNA repair, we next asked whether these agents could induce DNA damage. We exposed the cells to AZD1775 and / or AZD0156 for 24 hours, and then monitored DNA fragmentation at the single-cell level using a comet assay. We found that AZD1775 or AZD0156 alone promoted DNA damage compared with control, but both agents in combination caused markedly increased fragmentation, as indicated by comet tail intensity and moment (Fig. 3A and B). Consistent with this, western blot analysis revealed upregulated expression of the DNA damage marker $\gamma-\mathrm{H} 2 \mathrm{AX}$, with a greater effect observed in cells subjected to combination AZD1775 and AZD0156 treatment compared with monotherapy (Fig. 3C).

\section{Co-inhibition of WEE1 and ATM efficiently suppresses $P C$ cell migration}

To determine the consequences of WEE1 and ATM inhibition on PC cell function, we examined cell migration and invasion, which play well-characterized roles in cancer pro- gression. Importantly, very few studies have evaluated the effects of DDR-targeted agents on tumor cell migration. In this study, Transwell migration assay was employed. Interestingly, we found that cell migration and invasion of all four PC cell lines were significantly suppressed by treatment with $1 \mu \mathrm{M}$ AZD1775 or AZD0156 alone. However, the combination of both drugs resulted in efficiently inhibition of cell migration (Fig. 4A and B). Thus, WEE1 and ATM appear to have previously unrecognized functions in promoting PC cell migration.

The matrix metallopeptidases 9 and 2 (MMP-9 and MMP2) are known to be crucial for cancer metastasis and invasion $[17,18]$. Therefore, we asked whether the effects of WEE1 and / or ATM inhibition on PC cell migration were mediated via these enzymes. Western blot analysis revealed that treatment with AZD1775 or AZD0156 for 24 hours slightly decreased the expression of the smaller, active form of MMP9 compared with control cells, and this effect was augmented by co-treatment with both agents (Fig. 4C). In contrast, a reduction in active MMP-2 expression was only observed in combination-treated Capan-1 and SNU410 cells (Fig. 4C).

Next, we investigated the effects of DDR targeting on chemokine expression in Capan-1 cells using a human cytokine/ chemokine array. We found that the chemokines interleukin 8 (IL-8), CXCL1, CCL5, and CCL2 were significantly downregulated by AZD1775 treatment, combination therapy again showed an enhanced inhibitory effect on all four chemokines (Fig. 4D and E). Interestingly, previous work showed that signaling via the receptors for these chemokines (CXCR2, CCR5, and CCR2), facilitate the release of MMP-9, which contributes to enhanced angiogenesis and tumor metastasis [19-21]. Collectively, our results suggest that AZD1775 and AZD0156 act synergistically to suppress PC cell migration by reducing chemokine expression and MMP-9 release.

\section{Co-inhibition of WEE1 and ATM downregulates PD-L1 expression in PC cells}

Next, given that the pivotal status of PD-L1 expression in cancer cell or cancer microenvironment [22], we explored the potential interaction between the DDR and the anti-tumor immune response by examining the effects of DDR-targeted agents on total and cell surface expression of PD-L1 in PC cells. Western blot analysis revealed that total cellular PD-L1 expression was decreased by AZD1775 or AZD0156 treatment alone, but combination treatment was even more effective (Fig. 5A). This pattern was also observed when cell surface PD-L1 expression was examined by flow cytometry, particularly in SNU2913 cells, which express high PD-L1 levels (Fig. 5B).

To understand in more detail, the effects of WEE1 and ATM inhibition on PD-L1 expression, we investigated potential 

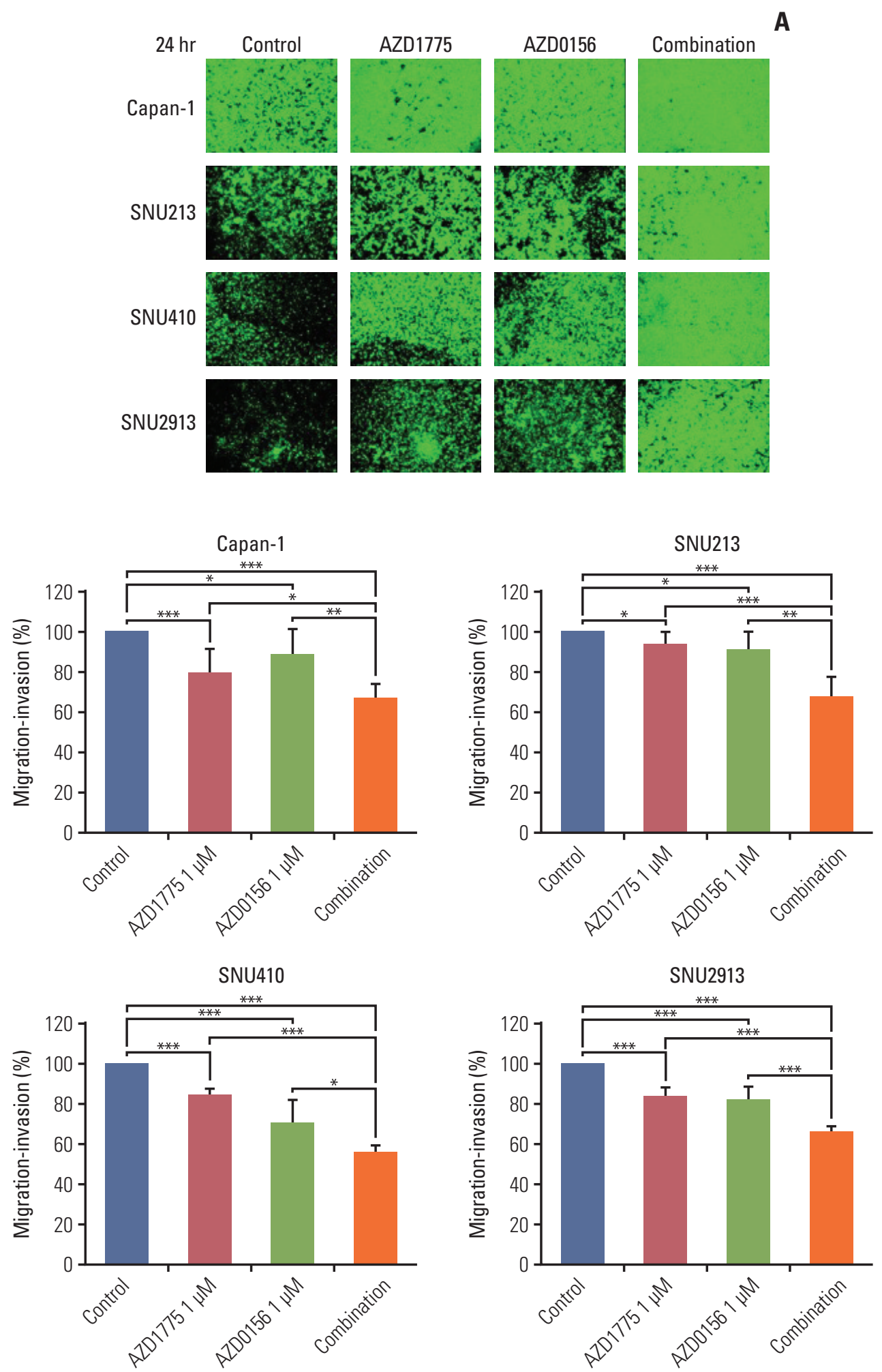

Fig. 4. Effect of WEE1 and ataxia telangiectasia mutated co-inhibition on migration of pancreatic cancer cells. (A) The antimigration effect was evaluated using cells with 0, AZD1775 $1 \mu \mathrm{mol} / \mathrm{L}$, or AZD0156 $1 \mu \mathrm{mol} / \mathrm{L}$ or both. The image was captured at 24 hours after treatment. (B) The percentage of migratory cells was analyzed after image was captured. The data represents three independent times. (Continued to the next page) 


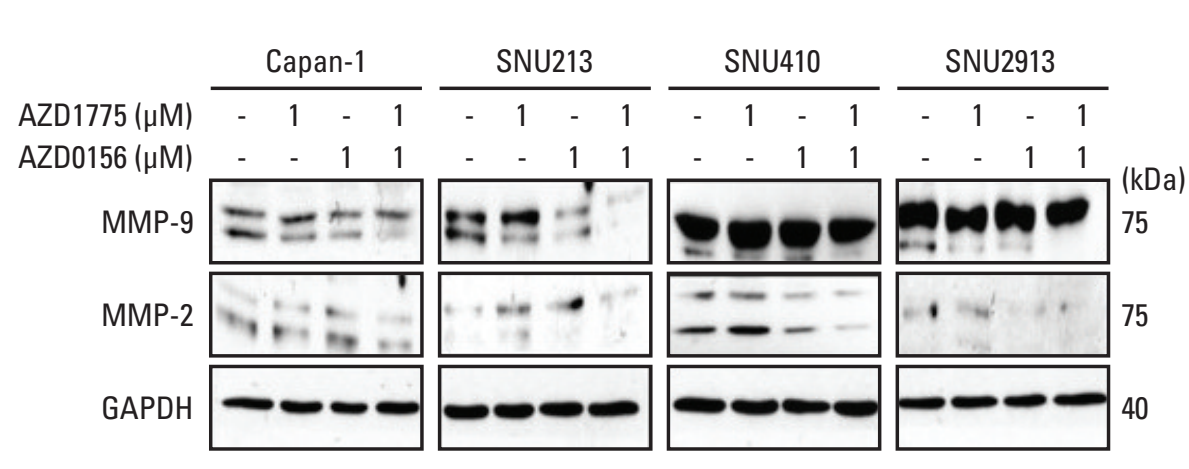

D
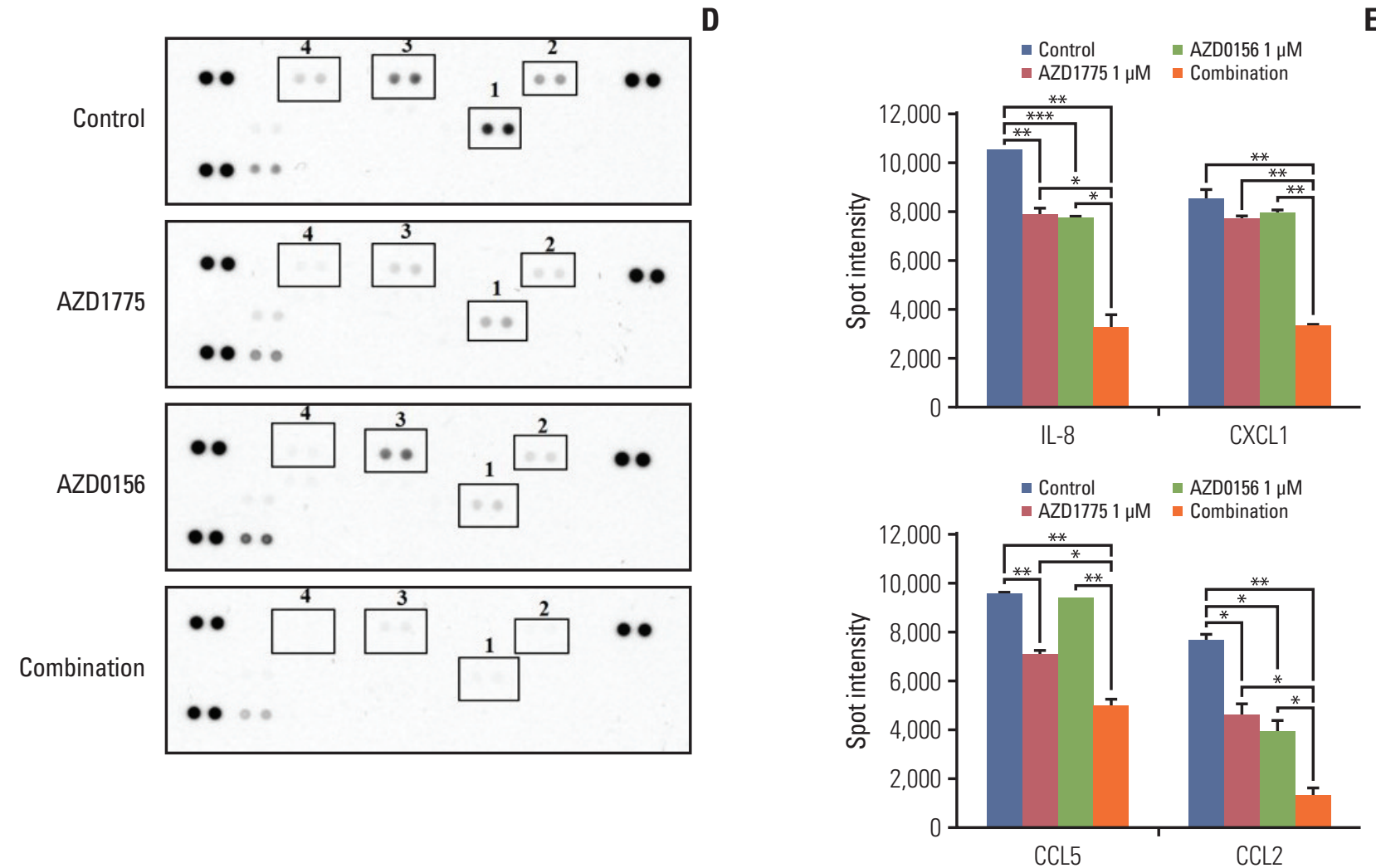

Fig. 4. (Continued from the previous page) (C) Western blot analysis of migration-related molecules in cells treated as described in panel A for 24 hours. MMP, matrix metallopeptidases; GAPDH, glyceraldehyde 3-phosphate dehydrogenase. (D, E) Human cytokine array analysis of Capan-1 cells treated as described for panel A for 24 hours. Spot intensities were quantified using ImageJ software. 1, interleukin 8 (IL-8); 2, CXCL1; 3, CCL5; 4, CCL2. ${ }^{*} \mathrm{p}<0.05,{ }^{* *} \mathrm{p}<0.01,{ }^{* * *} \mathrm{p}<0.001$.

mechanisms that regulate PD-L1: CMTM6, which is thought to prevent PD-L1 degradation by lysosomes [12], and GSK$3 \beta$, which has recently been identified as a novel regulator of PD-L1 expression [23]. Western blot analysis of CMTM6 expression showed a marked decrease in the protein levels after treatment for 72 hours with AZD1775 and AZD0156, either alone or in combination (Fig. 5A). To probe CMTM6mediated regulation of PD-L1 further, we asked whether this molecule is associated intracellularly by immunoprecipitat- ing with anti-PD-L1 and probing for the presence of CMTM6 in the immunoprecipitates. We found that CMTM6 was present in western blots of anti-PD-L1 immunoprecipitates, but not control IgG immunoprecipitates, of all four PC cell lines (Fig. 5C). Notably, immunoprecipitation (IP) with antiPD-L1 antibody showed the CMTM6-PD-L1 binding was decreased upon WEE1 or ATM inhibition, with the greatest effects observed upon dual inhibition. However, IP of CMTM6 followed by western blotting of PD-L1 did not show a 
$\infty$
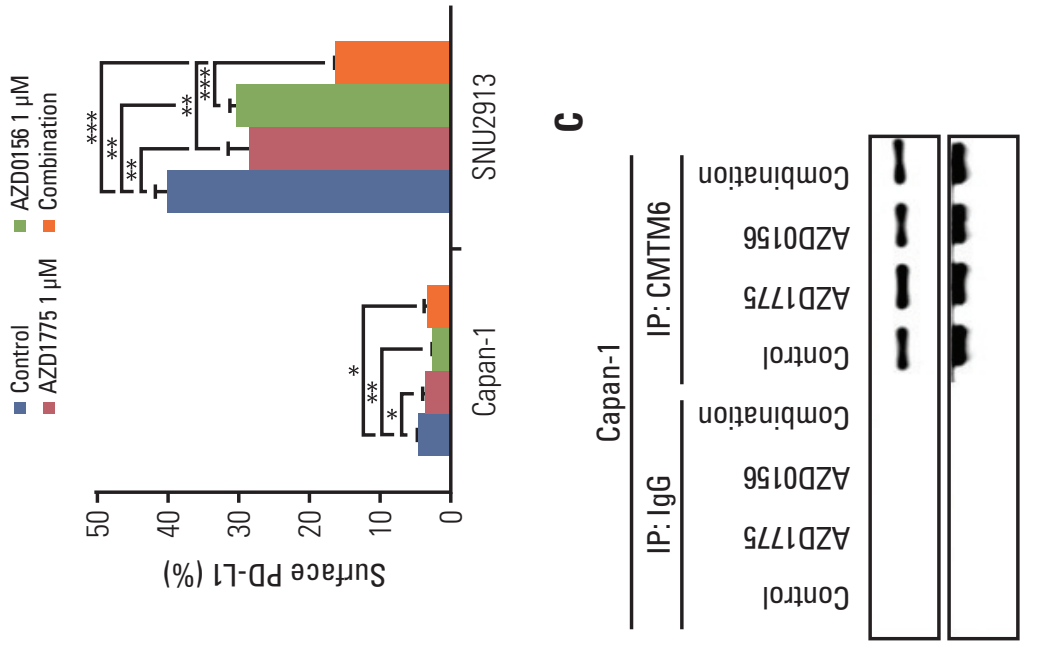

$\exists \frac{7}{0} \widehat{\theta}$

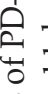

过

$\cdot \frac{\pi}{4}$ 岂

त्र

藏

응

कृ

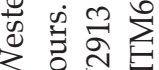

\ ำ

है क

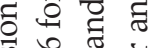

की เ๐

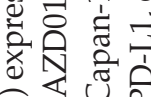

궁ㄷㅁ

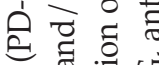

न 10 के

$\varangle$

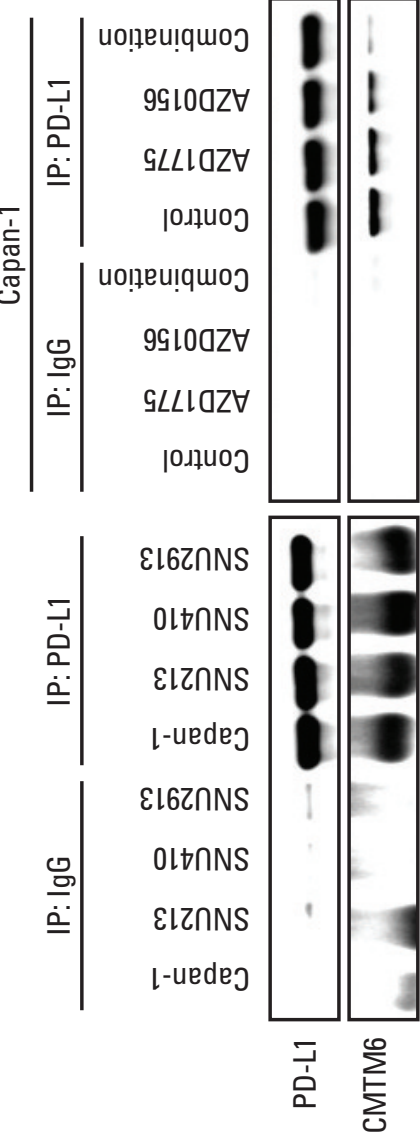

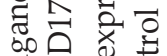

워

产宁
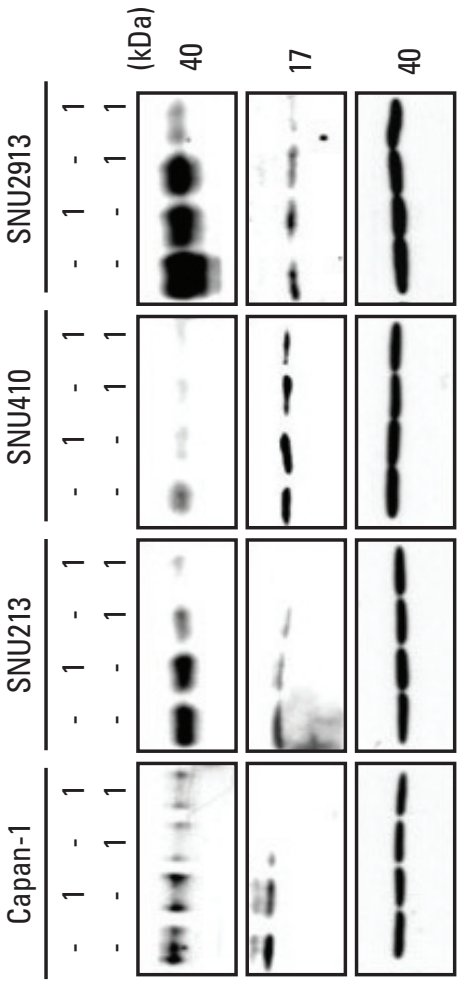

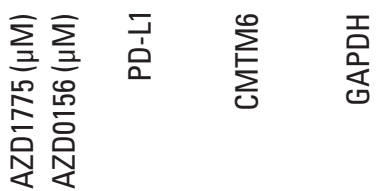

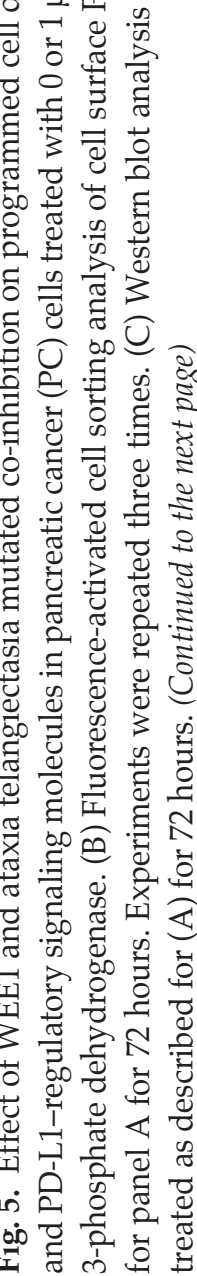


ш

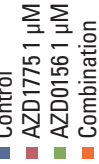

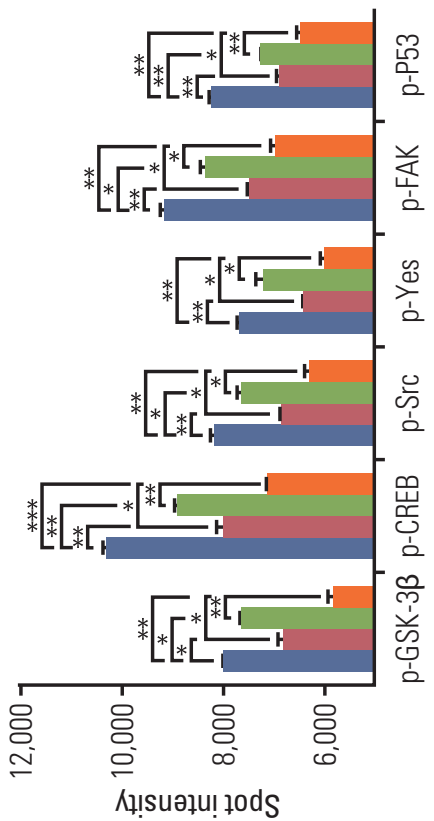

口

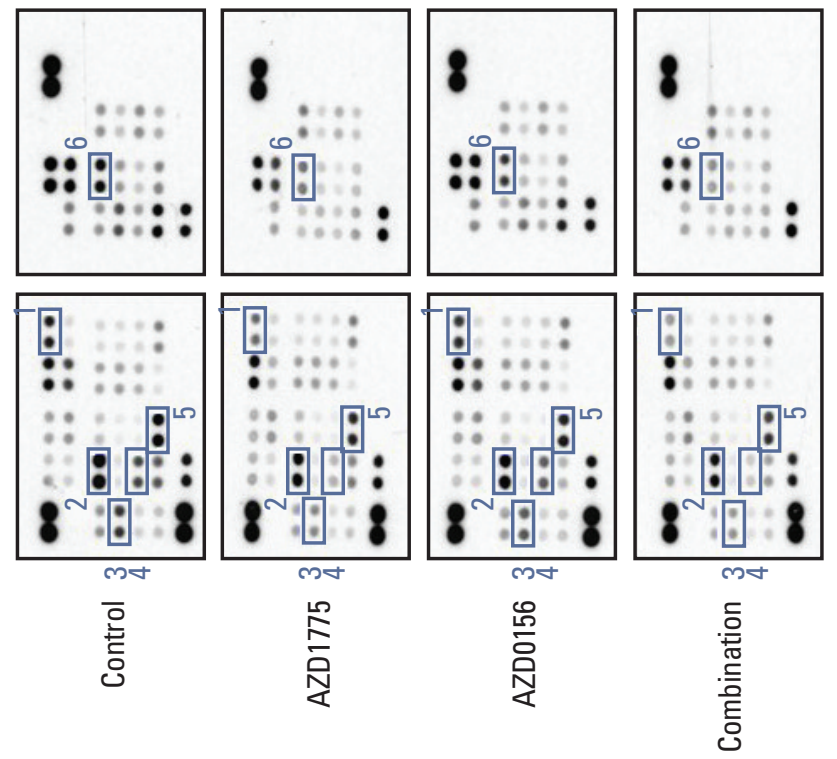

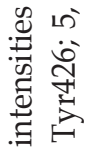

की है

के

实

휴

匹 के

巳

तี

क्षे

क $v$

突舆卷

$\tau$ i

ป

¿ V

๘ बัँ

क ले

U V

is

ㄷำ

ن

¿

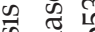

즐

ป $m$

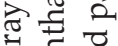

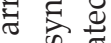

क

త్ ญี

궁

응 क

की पू

त

륭

है 至

式 क

II

다

ه

के तै

क 3

उ

ป

‡

ㅎำ

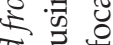

ป ర

记 考

పे है

ம்

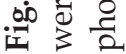


$\infty$

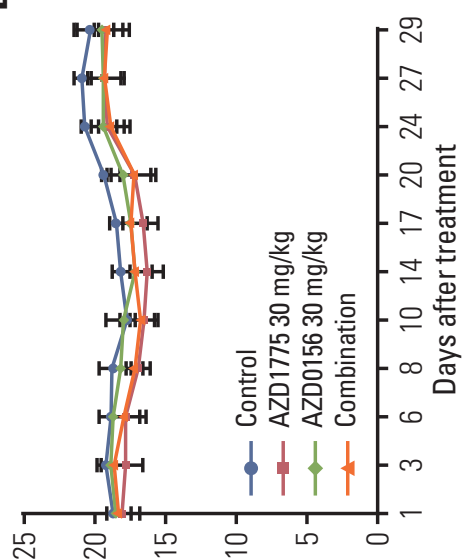

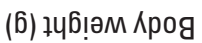

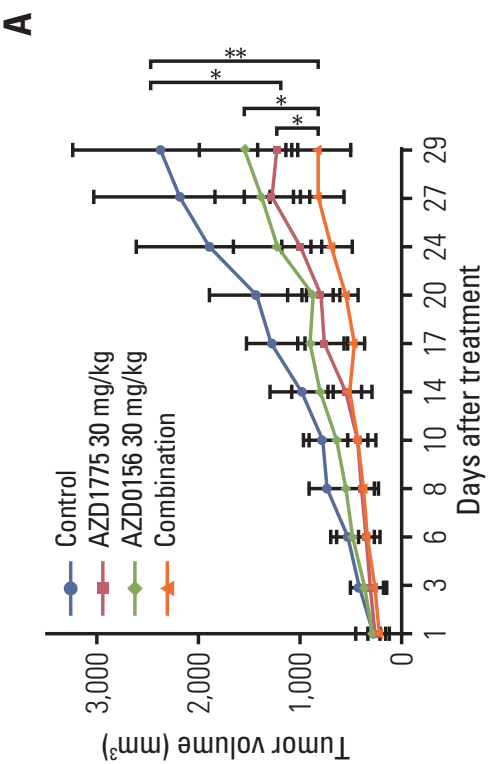

ט

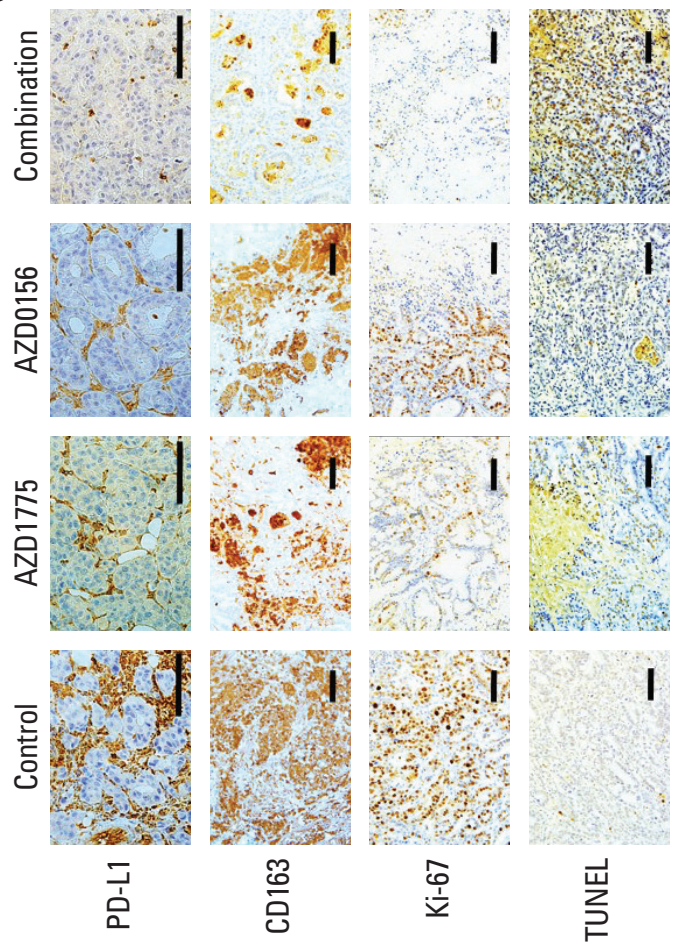

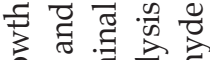

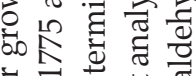

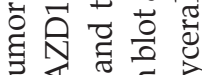

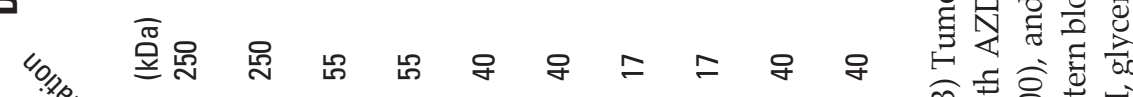

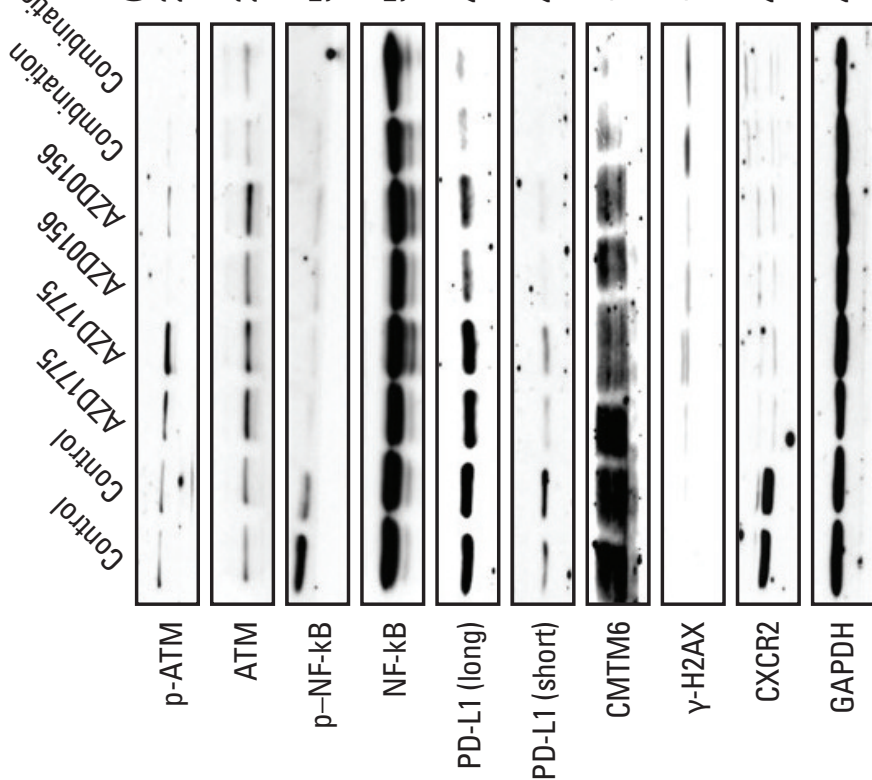

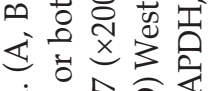

过

छ

范定 8

호 이 짐

ษ

。ั

考宁

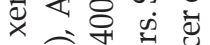

主雚艾

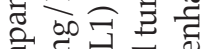

उ

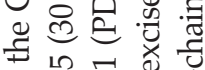

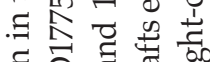

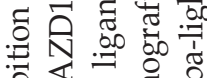

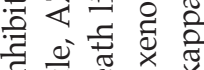

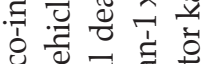

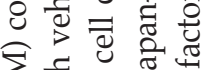

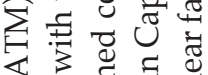

তั

䒕

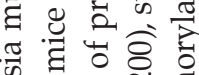

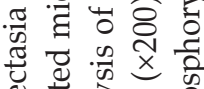

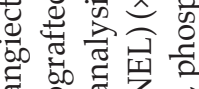

卷造考它

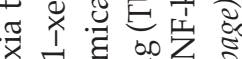

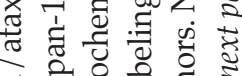

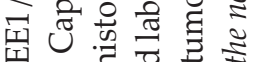

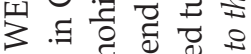

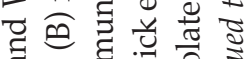

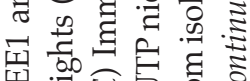

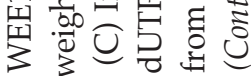

पे

पै

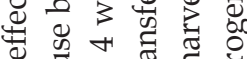

항요

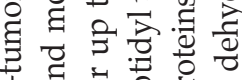

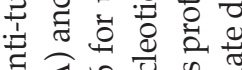

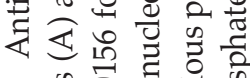

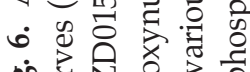

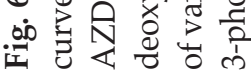



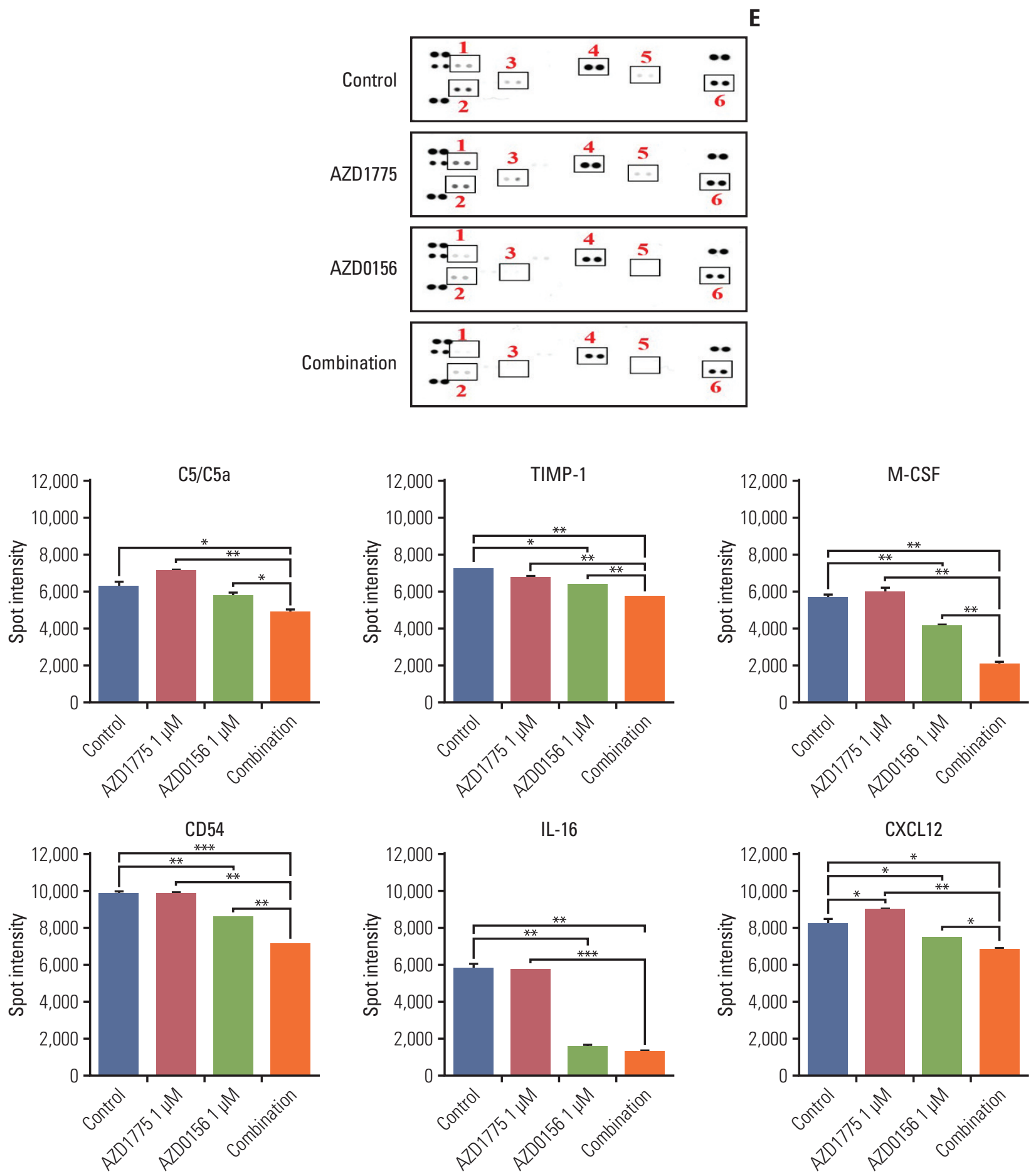

Fig. 6. (Continued from the previous page) (E, F) Mouse cytokine array analysis of serum collected from xenografted mice. Spot intensities were measured using ImageJ software. 1, C5/C5a; 2, TIMP metallopeptidase inhibitor (TIMP-1); 3, macrophage colony-stimulating factor (M-CSF); 4, CD54; 5, interleukin 16 (IL-16); 6, CXCL12. ${ }^{*} \mathrm{p}<0.05,{ }^{* *} \mathrm{p}<0.01,{ }^{* * *} \mathrm{p}<0.001$. 
comparable decrease in CMTM6-PD-L1 binding, even in cells co-treated with both AZD0156 and AZD1775 (Fig. 5C). It is possible that the reduction of CMTM6 by AZD1775 and AZD0156 led to decrease of CMTM6-PD-L1 bound form, which ultimately increase the PD-L1 degradation by lysosome supported by previous report [12].

To determine whether PD-L1 expression might be affected by the regulator GSK-3 $\beta$ in PC cells [23], we examined changes in phosphorylated kinase expression in AZD1775 and/or AZD0156-treated cells using a human phospho-kinase array. We observed that while AZD1775 and AZD0156 both downregulated $\mathrm{p}-\mathrm{GSK}-3 \beta$ expression, the combination treatment was more effective than either agent alone (Fig. 5D and E). This indicated that PD-L1 expression not only controlled by CMTM6 but also influenced by GSK-3 $\beta$ activity to a certain extent. Moreover, we found the same inhibitory effects on several other phospho-kinases, including phosphorylated cAMP response element-binding protein (p-CREB), p-Src, phosphorylated focal adhesion kinase (p-FAK), p-Yes, and p-p53 (Fig. 5D and E). Taken together, these results indicate that dual blockade of WEE1 and ATM may reduce PD-L1 expression by downregulating the expression of CMTM6, and inactive GSK-3 $\beta$.

\section{Co-inhibition of WEE1 and ATM augmented anti-tumor growth in Capan-1-xenograft model}

To confirm the anti-tumor effect of AZD1775 and AZD0156 in vivo, Capan-1 xenograft model was established. Despite high PD-L1 expression was detected in vitro in SNU2913 cells, since Capan-1 was more tumorigenic than SNU2913, we selected Capan-1 xenograft models to conduct the following experiments. Consistent with the in vitro observations, we found that single-agent treatment with AZD1775 or AZD0156 significantly decreased tumor growth, but the combination treatment was markedly more effective than either agent alone $(\mathrm{p}<0.05)$ (Fig. 6A). We observed no overt evidence of drug toxicity, as reflected by a lack of significant change in body weight (Fig. 6B).

Next, we excised the tumors and performed immunohistochemical (IHC) staining of various markers. As we had observed in vitro, PD-L1 expression was decreased by AZD1775 or AZD0156 monotherapy and decreased by combination treatment (Fig. 6C). Moreover, CD163, an M2-type macrophage marker, was expressed at high levels in tumors from control mice but at lower levels in tumors from AZD1775- and/or AZD0156-treated mice (Fig. 6C). Combination treatment with both drugs also potently inhibited staining of the proliferation marker Ki-67 and increased terminal deoxynucleotidyl transferase dUTP nick end labeling staining, which detects fragmented DNA (Fig. 6C). In parallel, we performed western blot analysis to confirm our in vitro findings in the isolated tumors. In line with the in vitro results, we confirmed that AZD1775 treatment upregulated p-ATM while combination treatment with AZD-1775 and the ATM inhibitor reversed this. Moreover, the expression of p-NF-kB and CXCR2 were profoundly blocked by AZD1775 and/or AZD0156 treatment, and AZD1775 plus AZD0156 enhanced $\gamma-\mathrm{H} 2 \mathrm{AX}$ accumulation. Furthermore, we validated the in vitro findings with PD-L1 and CMTM6 by confirming that both proteins were downregulated by single- or dualagent treatment (Fig. 6D).

Finally, we assessed the effects of the DDR-targeting agents on immune cell activity by analyzing cytokine levels in the sera of tumor-bearing mice. Using a mouse cytokine array, we found that release of C5a, TIMP metallopeptidase inhibitor 1 (TIMP-1), macrophage colony-stimulating factor, CD54, IL-16, and CXCL12 were markedly reduced by AZD1775 and /or AZD0156 treatment, with larger effects observed in drug combination-treated mice (Fig. $6 \mathrm{E}$ and F). Collectively, these in vivo data confirm that dual targeting of the DDR pathway components WEE1 and ATM profoundly suppress tumor growth in vivo compared with blockade of either molecule alone.

\section{Discussion}

Although ATM is a tumor suppressor gene, both of WEE1 and ATM promote DNA repair to maintain cell survival during the progression of the cancers [24]. Inhibition of WEE1 and ATM activity are our opportunity to kill the cancers by facilitating DNA damage.

In this study, we demonstrate for the first time that targeting the DDR by inhibition of WEE1 has anti-tumor effects in PC and that co-inhibition of WEE1 and ATM amplifies these effects. We also identified crosstalk between the DDR pathway and immune system by demonstrating DDR-dependent regulation of PD-L1 expression in PC cells.

One of the main findings of this study is that dual inhibition of WEE1 and ATM has profound effects on PC cell migration. Only a few studies have been performed on the anti-migratory effects of DDR-acting agents. One report showed that AZD1775 inhibits the migration of gastric cancer cells, although the mechanism of action was not elucidated [25]. Another study suggested that ATM might promote cell migration by regulating IL-8 expression independently of its role in DNA double-strand break repair [26]. We found that WEE1 and ATM co-inhibition reduced the expression of MMP-9, IL-8, CXCL1, CCL2, and CCL5, which is consistent with an anti-migratory effect. This is the first demonstration of the involvement of WEE1 or ATM in MMP-9 or IL-8 reg- 
ulation. Moreover, downregulation of CXCR2, which is the IL-8 and CXCL1 receptor, in PC tumors was confirmed in our in vivo experiments. Although CXCR2 inhibition was shown to profoundly suppress metastasis and augments anti-PD-1 therapy in PC [10], the current study is the first to examine CXCR2 expression in PC. Since CXCR2 and CCR2 are also expressed by M2 macrophages, we speculate that combination AZD1775 and AZD0156 treatment might impair M2 type polarization [27]. In addition, the involvement of Src and FAK in promoting cell migration and invasion is well known [28], and a recent report implicates a similar role for $\mathrm{p}$-CREB [29]. Thus, our finding that expression of p-CREB, p-Src, p-Yes, and p-FAK are profoundly inhibited by WEE1 and ATM co-inhibition is consistent with a role for these molecules in PC cell migration. Prior to this study, there had been no reports of a relationship between Src/FAK/CREB and the DDR pathway in PC.

An interesting recent study reported that PARP inhibition upregulates PD-L1 expression in breast cancer via inactivation of GSK-3 $\beta$ [16]. We also found a similar increase in PD-L1 expression in PARP inhibitor (Olaparib)-treated PC cells (data not shown). In contrast, the present study showed here that targeting of the DDR by WEE1 and / or ATM inhibition reduced PD-L1 expression concomitantly with downregulation of p-GSK-3 $\beta$ Ser9 level, the inactive form of GSK$3 \beta$. This mechanism might at least partly explain the reduction in PD-L1 induced by WEE1 and / or ATM inhibition. It is interesting to note that PARP, WEE1, and ATM have distinct effects on PD-L1 expression, despite the fact that they are all core members of the DDR signaling network. Thus, while PARP inhibition increases PD-L1 expression by enhancing GSK-3 $\beta$ inactivation, WEE1 and/or ATM inhibition does the opposite by decreasing the expression of inactive GSK-3 $\beta$.

Among the mechanisms known to regulate PD-L1 expression, many act at the transcriptional level, including the JAKSTAT pathway, c-Myc, and NF-кB $[15,30,31]$. Consistent with this literature, we also observed a reduction in P-STAT- 1 and p-NF-kB concomitant with PD-L1 downregulation in WEE1 and/or ATM inhibitor-treated cells. More recently, Burr and colleagues used CRISPR/Cas9 technology to screen approximately 20,000 genes in the human PC cell line BxPC-3, and they identified a novel protein, CMTM6, as a major regulator of PD-L1 expression [12]. Importantly, we observed that CMTM6 binds to PD-L1 in PC cells and that CMTM6 expression was reduced upon inhibition of WEE1 and/or ATM. As mentioned earlier, CMTM6 could rescue and recycle the PD-L1 from the lysosome to the cell surface. More recently, an interesting study revealed that HIP1R facilitates the delivery of PD-L1 to the lysosome [32]. Here, we speculated two potential mechanisms of the CMTM6 reduction by WEE1 or ATM. First of all, WEE1 or ATM might post-transcriptionally increase the HIP1R expression and compete for CMTM6 to bind with PD-L1. Secondly, CMTM6 might be transcriptionally downregulated by WEE1 or ATM, and these events further disrupt the interaction between PD-L1 and CMTM6. The mechanism by which DDR targeting blocks CMTM6 expression remains to be clarified.

As mentioned earlier, KRAS and TP53 are frequently mutated in PC. Recent work suggests that KRAS mutant cancer cells can enhance the recruitment of MDSCs and M2 macrophages to the tumor, thereby contributing to evasion of the immune response. M2 macrophages can also be recruited by cancer cells carrying mutant TP53 [9]. These macrophages promote tumor evasion through multiple mechanisms, including enhanced angiogenesis and tissue remodeling, recruitment of MDSCs and CAFs, and inhibition of T cell function and proliferation, thus enhancing metastasis and invasion [33]. Here, we evaluated M2 macrophage recruitment to the tumor in the mouse xenograft model by IHC staining of CD163, and we found that the high basal levels of tumor-associated $\mathrm{CD} 163^{+}$cells were markedly decreased by AZD1775 and/or AZD0156 treatment. This finding supports the possibility that combination therapy suppresses recruitment of M2 macrophages to the tumor, and it will be of interest to understand how other cells in the tumor microenvironment are affected by DDR-targeting agents.

We used high concentrations of WEE1 inhibitor (AZD1775$1 \mu \mathrm{M}$ ) and ATM inhibitor (AZD0156 $1 \mu \mathrm{M}$ ) in current in vitro experiments considering the active pharmacology of compounds. Particularly, AZD0156 $1 \mu \mathrm{M}$ might have off-target effects.

Taken together, our results demonstrate that DDR-targeting agents such as WEE1 and ATM inhibitors have potent, synergistic anti-tumor effects in PC. We also identified crosstalk between the DDR and immune response in PC, since WEE1/ATM inhibition downregulates CXCR2, CD163, and PD-L1 expression, the latter by blocking expression of inactive $\mathrm{p}-\mathrm{GSK}-3 \beta$ and CMTM6. The findings in this study support further clinical development of DDR-targeting strategies for the treatment of PC.

\section{Electronic Supplementary Material}

Supplementary materials are available at Cancer Research and Treatment website (https:// www.e-crt.org).

\section{Conflicts of Interest}

D-Y Oh received AstraZeneca-KHIDI (Korea Health Industry Development Institute) Oncology Research Program and Y-J Bang is a consultant/advisory board member of AstraZeneca. No conflicts of interest were disclosed by the other authors. 


\section{Acknowledgments}

This research was supported by 3rd AstraZeneca-KHIDI (Korea Health Industry Development Institute) oncology research program (grant No. 06-2016-2920), Research Resettlement Fund for the new faculty of Seoul National University (grant No. 800-2016-0312) and Research fund from SNUH (grant No. 03-2017-0100).

\section{References}

1. Bailey P, Chang DK, Nones K, Johns AL, Patch AM, Gingras $\mathrm{MC}$, et al. Genomic analyses identify molecular subtypes of pancreatic cancer. Nature. 2016;531:47-52.

2. Choi Y, Oh DY, Kim K, Chie EK, Kim TY, Lee KH, et al. Concurrent chemoradiotherapy versus chemotherapy alone for unresectable locally advanced pancreatic cancer: a retrospective cohort study. Cancer Res Treat. 2016;48:1045-55.

3. O'Connor MJ. Targeting the DNA damage response in cancer. Mol Cell. 2015;60:547-60.

4. Richer AL, Cala JM, O'Brien K, Carson VM, Inge LJ, Whitsett TG. WEE1 kinase inhibitor AZD1775 has preclinical efficacy in LKB1-deficient non-small cell lung cancer. Cancer Res. 2017;77:4663-72

5. Kaufman B, Shapira-Frommer R, Schmutzler RK, Audeh MW, Friedlander M, Balmana J, et al. Olaparib monotherapy in patients with advanced cancer and a germline BRCA1/2 mutation. J Clin Oncol. 2015;33:244-50.

6. Chen CC, Kass EM, Yen WF, Ludwig T, Moynahan ME, Chaudhuri J, et al. ATM loss leads to synthetic lethality in BRCA1 BRCT mutant mice associated with exacerbated defects in homology-directed repair. Proc Natl Acad Sci U S A. 2017; 114:7665-70.

7. Mohni KN, Kavanaugh GM, Cortez D. ATR pathway inhibition is synthetically lethal in cancer cells with ERCC1 deficiency. Cancer Res. 2014;74:2835-45.

8. Matheson CJ, Backos DS, Reigan P. Targeting WEE1 kinase in cancer. Trends Pharmacol Sci. 2016;37:872-81.

9. Binnewies M, Roberts EW, Kersten K, Chan V, Fearon DF, Merad M, et al. Understanding the tumor immune microenvironment (TIME) for effective therapy. Nat Med. 2018;24:54150.

10. Steele CW, Karim SA, Leach JD, Bailey P, Upstill-Goddard R, Rishi L, et al. CXCR2 inhibition profoundly suppresses metastases and augments immunotherapy in pancreatic ductal adenocarcinoma. Cancer Cell. 2016;29:832-45.

11. Chen DS, Mellman I. Oncology meets immunology: the cancer-immunity cycle. Immunity. 2013;39:1-10.

12. Burr ML, Sparbier CE, Chan YC, Williamson JC, Woods K, Beavis PA, et al. CMTM6 maintains the expression of PD-L1 and regulates anti-tumour immunity. Nature. 2017;549:101-5.

13. Chatzinikolaou G, Karakasilioti I, Garinis GA. DNA damage and innate immunity: links and trade-offs. Trends Immunol. 2014;35:429-35.

14. Rosado MM, Bennici E, Novelli F, Pioli C. Beyond DNA repair, the immunological role of PARP-1 and its siblings. Immunol- ogy. 2013;139:428-37.

15. Sato H, Niimi A, Yasuhara T, Permata TB, Hagiwara $Y$, Isono $\mathrm{M}$, et al. DNA double-strand break repair pathway regulates PD-L1 expression in cancer cells. Nat Commun. 2017;8:1751.

16. Jiao S, Xia W, Yamaguchi H, Wei Y, Chen MK, Hsu JM, et al. PARP inhibitor upregulates PD-L1 expression and enhances cancer-associated immunosuppression. Clin Cancer Res. 2017; 23:3711-20.

17. Farina AR, Mackay AR. Gelatinase B/MMP-9 in tumour pathogenesis and progression. Cancers (Basel). 2014;6:240-96.

18. Gialeli C, Theocharis AD, Karamanos NK. Roles of matrix metalloproteinases in cancer progression and their pharmacological targeting. FEBS J. 2011;278:16-27.

19. Zarogoulidis P, Katsikogianni F, Tsiouda T, Sakkas A, Katsikogiannis N, Zarogoulidis K. Interleukin-8 and interleukin-17 for cancer. Cancer Invest. 2014;32:197-205.

20. Chuang JY, Yang WH, Chen HT, Huang CY, Tan TW, Lin YT, et al. CCL5/CCR5 axis promotes the motility of human oral cancer cells. J Cell Physiol. 2009;220:418-26.

21. Tang CH, Tsai CC. CCL2 increases MMP-9 expression and cell motility in human chondrosarcoma cells via the Ras/Raf/ MEK/ERK/NF-KB signaling pathway. Biochem Pharmacol. 2012;83:335-44.

22. Topalian SL, Drake CG, Pardoll DM. Immune checkpoint blockade: a common denominator approach to cancer therapy. Cancer Cell. 2015;27:450-61.

23. Li CW, Lim SO, Xia W, Lee HH, Chan LC, Kuo CW, et al. Glycosylation and stabilization of programmed death ligand-1 suppresses T-cell activity. Nat Commun. 2016;7:12632.

24. Pilie PG, Tang C, Mills GB, Yap TA. State-of-the-art strategies for targeting the DNA damage response in cancer. Nat Rev Clin Oncol. 2019;16:81-104.

25. Chen D, Lin X, Gao J, Shen L, Li Z, Dong B, et al. Wee1 inhibitor AZD1775 combined with cisplatin potentiates anticancer activity against gastric cancer by increasing DNA damage and cell apoptosis. Biomed Res Int. 2018;2018:5813292.

26. Chen WT, Ebelt ND, Stracker TH, Xhemalce B, Van Den Berg CL, Miller KM. ATM regulation of IL-8 links oxidative stress to cancer cell migration and invasion. Elife. 2015;4:e07270.

27. Dalton HJ, Armaiz-Pena GN, Gonzalez-Villasana V, LopezBerestein G, Bar-Eli M, Sood AK. Monocyte subpopulations in angiogenesis. Cancer Res. 2014;74:1287-93.

28. Kolli-Bouhafs K, Sick E, Noulet F, Gies JP, De Mey J, Ronde P. FAK competes for Src to promote migration against invasion in melanoma cells. Cell Death Dis. 2014;5:e1379. 
29. Zhang ZX, Zhang WN, Sun YY, Li YH, Xu ZM, Fu WN. CREB promotes laryngeal cancer cell migration via MYCT1/ NAT10 axis. Onco Targets Ther. 2018;11:1323-31.

30. Garcia-Diaz A, Shin DS, Moreno BH, Saco J, Escuin-Ordinas $\mathrm{H}$, Rodriguez GA, et al. Interferon receptor signaling pathways regulating PD-L1 and PD-L2 expression. Cell Rep. 2017; 19:1189-201.

31. Asgarova A, Asgarov K, Godet Y, Peixoto P, Nadaradjane A,
Boyer-Guittaut M, et al. PD-L1 expression is regulated by both DNA methylation and NF-kB during EMT signaling in nonsmall cell lung carcinoma. Oncoimmunology. 2018;7:e1423170.

32. Wang H, Yao H, Li C, Shi H, Lan J, Li Z, et al. HIP1R targets PD-L1 to lysosomal degradation to alter T cell-mediated cytotoxicity. Nat Chem Biol. 2019;15:42-50.

33. Aras S, Zaidi MR. TAMeless traitors: macrophages in cancer progression and metastasis. Br J Cancer. 2017;117:1583-91. 